\title{
Research Note \\ On the relation between the IR continuum and the active galactic nucleus in Seyfert galaxies ${ }^{\star}$
}

\author{
M. A. Prieto ${ }^{1,2}$, A. M. Pérez García ${ }^{2}$, and J. M. Rodríguez Espinosa ${ }^{2}$ \\ 1 European Southern Observatory, 85748 Garching, Germany \\ 2 Instituto de Astrofísica de Canarias, 38200 La Laguna (Tenerife), Spain
}

Received 15 November 2000 / Accepted 26 July 2001

\begin{abstract}
A sample of the brightest known Seyfert galaxies from the CfA sample is analyzed on the basis of ISO photometric and spectroscopic data. Regardless of the Seyfert type, the mid-IR continuum emission from these galaxies is found to be correlated with the coronal line emission arising in the nuclear active region. Conversely, the correlation degrades progressively when moving from the mid- to the far-IR emission, where it vanishes. It is concluded that the mid-IR emission is largely dominated by dust heated by processes associated with the active nucleus whereas the far-IR is a different component most probably unrelated with the active region. We suggest that the far-IR component is due to dust heated by the stellar population in the disks of these galaxies.
\end{abstract}

Key words. galaxies: Seyfert - infrared: galaxies

\section{Introduction}

Dust reprocessing of some primary emission is at present regarded as the most likely source for the mid- and farIR emission in Seyfert galaxies. Whether dust is predominantly heated by starlight, processes associated with the central power source - photoionization, shocks - or a mixture thereof may depend on the region of the IR spectrum under study. The mid-IR emission was found by Edelson et al. (1987) to be extended on scales of about $1 \mathrm{kpc}$ in Seyfert $2 \mathrm{~s}$ and many Seyfert $1 \mathrm{~s}$, and therefore most probably related to dust heated by star forming regions. This result has been corroborated by several authors (Giuricin et al. 1995; Maiolino et al. 1995) using much larger samples of Seyfert galaxies. Regarding the far-IR, RodriguezEspinosa et al. (1986) concluded that a large fraction of the IRAS far-IR emission in Seyfert's is due to dust heated by extended starforming events. This is also confirmed by Giuricin et al. (1995) and Rigopoulou et al. (1997) among others mainly on the basis of IRAS data. More recently, Pérez García et al. (1998) and Pérez García \& Rodríguez Espinosa (2001) have shown on the basis of ISOPHOT data that the spectral energy distribution out to $200 \mu \mathrm{m}$

\footnotetext{
Send offprint requests to: A. M. Pérez García, e-mail: apg@ll.iac.es

* Based on observations with ISO, an ESA project with instruments funded by ESA Member States (especially the PI countries: France, Germany, The Netherlands and the UK) and with the participation of ISAS and NASA.
}

of Seyfert galaxies is of thermal origin and the sum of three different components: a warm component peaking at about $16 \mu \mathrm{m}$, related with the circumnuclear and nuclear emission; a cold component peaking at $\sim 60 \mu \mathrm{m}$, produced by dust heated by starforming regions in the host galaxy; and a very cold component peaking at $\sim 150 \mu \mathrm{m}$, emitted by dust heated by the general interstellar radiation field. These authors further show that the far-IR emission in those objects for which ISOPHOT $90 \mu \mathrm{m}$ maps are available, can be traced out to an extension which is similar or larger than that seen in optical $R$ band images (Pérez García et al. 2000).

In this work, we would like to further examine the nature of the aforementioned IR components, in particular, in regard to the contribution of the central AGN source. In doing so, the ISO continuum emission of a sample of very bright Seyfert galaxies is compared with a reliable tracer of the AGN activity, that is their coronal line spectrum. Coronal lines are unique tracers of the AGN activity, the reason being the high ionization potential of the species that give origin to them. These lines form in very energetic environments, because of their high ionization potential they are produced very close to the excitation source, thus tracing a rather compact region close to the central AGN source. The coronal lines used in this work require photon energies in the $50-300 \mathrm{eV}$ range and thus their origin is directly linked to processes associated with the AGN. In principle, the main dust heater in the nuclear region are both circumnuclear star forming regions 
and the active nucleus. Clues on which of those mechanisms is predominant, and in turn to probe into the AGN energetics may be found by comparing the coronal line spectra with the IR continuum emission. The relationship between these two independent energy nuclear tracers is thus investigated herein.

\section{The Seyfert sample used in this work}

The sample of galaxies used in this work is extracted from the CfA sample of Seyfert galaxies. The complete CfA sample was observed with ISOPHOT in the wavelength range between 16 and $200 \mu \mathrm{m}$. The brightest members were the subject of a complementary spectroscopic study with the ISO short wavelength spectrometer ISOSWS. This spectroscopic sample includes all the CfA Seyferts detected with IRAS at $12 \mu \mathrm{m}$ and is the one used in this work. It is further complemented with the Southern Circinus galaxy which shows one the brightest ISO coronal spectra known (Moorwood et al. 1996). For all these galaxies, observations of the coronal lines [O IV] $25.9 \mu \mathrm{m}$, [Ne V] $14.3 \mu \mathrm{m},[\mathrm{Mg}$ VIII] $3.02 \mu \mathrm{m}$ and [Si IX] $2.58 \mu \mathrm{m}$ were obtained. The reader is referred to Pérez García et al. (1998) and to Prieto \& Viegas (2000) for further details regarding the observations and analysis of the ISOPHOT and ISOSWS data respectively.

In total, the sample contains five Seyfert type 1 and seven type 2. All the galaxies present strong $[\mathrm{O} \quad \mathrm{IV}] \lambda 25.9 \mu \mathrm{m}$ and $[\mathrm{Ne} \mathrm{V}] \lambda 14.3 \mu \mathrm{m}$ lines whereas $[\mathrm{Mg} \mathrm{VIII}] \lambda 3.02 \mu \mathrm{m}$ and [Si IX] $\lambda 2.58 \mu \mathrm{m}$ are systematically the weakest lines (cf. Table 1 in Prieto \& Viegas 1999). Clear detections for the later are only found in Circinus, NGC 1068, NGC 4151, and possibly in Mrk 817. Due to the weakness of the $[\mathrm{Mg}$ VIII $] \lambda 3.02 \mu \mathrm{m}$ and [Si IX] $\lambda 2.58 \mu \mathrm{m}$ lines, the present analysis is based on the $[\mathrm{O}$ IV $] \lambda 25.9 \mu \mathrm{m}$ and $[\mathrm{Ne} \mathrm{V}] \lambda 14.3 \mu \mathrm{m}$ data only. The fluxes for these lines correspond to integrated values within the ISO-SWS aperture of $20 \times 33$ arcsecs. Details of these observations can be found in Prieto \& Viegas (2000).

The ISOPHOT data covers the $16-200 \mu \mathrm{m}$ range. Continuum fluxes at 16, 25, 60, 90, 120, 135, 180 and $200 \mu \mathrm{m}$ were measured for all the galaxies in this sample. In addition, ground based data at $10 \mu \mathrm{m}$ taken from Contini et al. (1999), Edelson (1978), Rieke \& Lebovsky (1978), Edelson et al. (1987), Maiolino et al. (1995) and Maiolino et al. (1998) are used. These correspond to integrated fluxes within an aperture between 5 and 8.5 arcsec. The 16 and $25 \mu \mathrm{m}$ values correspond to integrated fluxes within a 120 or 180 arcsec aperture, depending on the size of the objects. The 60 and $90 \mu \mathrm{m}$ data were acquired with the $\mathrm{C} 100$ detector consisting of a $3 \times 3$ pixel array, each pixel projecting on $45 \operatorname{arcsec}$ on the sky. The longer wavelengths observations were done with a $2 \times 2$ pixel array (C200), each pixel projecting on 89.9 arcsec on the sky. Although apertures for the mid- and far- IR fluxes are different, in all cases for each galaxy the integrated fluxes include the entire object. Details of these observations are in Pérez García et al. (1998).
All the ISO fluxes used in this work are well above the detection limit of our observations with ISOPHOT and ISOSWS. Our detection limit with ISOPHOT is about $50 \mathrm{mJy}$ at 16 and $25 \mu \mathrm{m}$. This is estimated on the basis of the ISOPHOT observations of the complete CfA sample of galaxies. At 60 and $90 \mu \mathrm{m}$, the present sample is far above the ISO detection limit: the minimum flux used in this work is above $200 \mathrm{mJy}$. In the case of the ISOSWS, our detection limit is about $10^{-21} \mathrm{~W} \mathrm{~cm}^{-2}$ in the spectral regions around the $[\mathrm{OIV}]$ and $[\mathrm{NeV}]$ lines. This is consistent with the minimum line fluxes detected with good confidence by Genzel et al. (1998) of a few $10^{-21} \mathrm{~W} \mathrm{~cm}^{-2}$.

\section{Results}

Figures 1 and 2 show various comparisons between the [O IV] $\lambda 25.9 \mu \mathrm{m}$ and [Ne V] $\lambda 14.3 \mu \mathrm{m}$ coronal lines fluxes and the 10,16, 25, 60 and $90 \mu \mathrm{m}$ continuum fluxes for all the galaxies in the sample. In all the figures, the brightest objects in the diagrams are by decreasing flux level N1068, Circiunus and NGC 4151.

Focusing first on the mid-IR continuum emission, a correlation is clearly seen between the coronal line emission and the mid IR $10 \mu \mathrm{m}$ (Fig. 1, top panels), 16 and $25 \mu \mathrm{m}$ continuum fluxes (Fig. 2 top two panels). The linear correlation coefficient is $r=0.98$ for the comparison of the $10 \mu \mathrm{m}$ flux with both the $[\mathrm{NeV}]$ and [OIV] lines, implying a probability of chance occurrence in a uncorrelated parent population of less than $0.1 \%$. The significance of the correlations decreases as the comparison involves longer wavelength continuum fluxes. This may be partially due to the fact that the $10 \mu \mathrm{m}$ emission is collected from a much small aperture than the ISO apertures and hence, it should be less polluted by circumnuclear star formation. Formally, the comparison with the $16 \mu \mathrm{m}$ flux yields linear correlation coefficients of $r=0.86$ and $r=0.92$ for the $[\mathrm{Ne} \mathrm{V}]$ and the [OIV] lines respectively $(0.1 \%$ of chance occurrence). In general, the correlation with [O IV] $25.9 \mu \mathrm{m}$ is better as this line is the strongest and best measured in the ISOSWS spectra (Prieto \& Viegas 2000).

The comparison with the $25 \mu \mathrm{m}$ continuum flux shows a correlation slightly weaker than that seen with the shorter wavelengths IR fluxes. The correlation coefficients are $r=0.81$ and $r=0.87$ for the $[\mathrm{Ne} \mathrm{V]}$ and the [OIV] lines respectively, with a significance level of $99 \%$.

When moving to much cooler continuum fluxes, i.e., beyond $60 \mu \mathrm{m}$ (Fig. 2 bottom two panels), the distribution of points becomes rather scattered - note that all panels in Figs. 1 and 2 have the same scale. The scatter increases with increasing IR wavelength, this trend holding up to the longest IR wavelength traced with ISO, about $200 \mu \mathrm{m}$ (note that to avoid redundancy, only the comparisons with the fluxes at 60 and $90 \mu \mathrm{m}$ are shown). The correlation coefficients are accordingly lower than those derived with the short wavelength IR fluxes. For example, the linear correlation coefficient between the $60 \mu \mathrm{m}$ emission and the [OIV] line is 0.7 and between the $90 \mu \mathrm{m}$ and the [OIV] line is $0.6-98 \%$ significance level. This result is compatible 

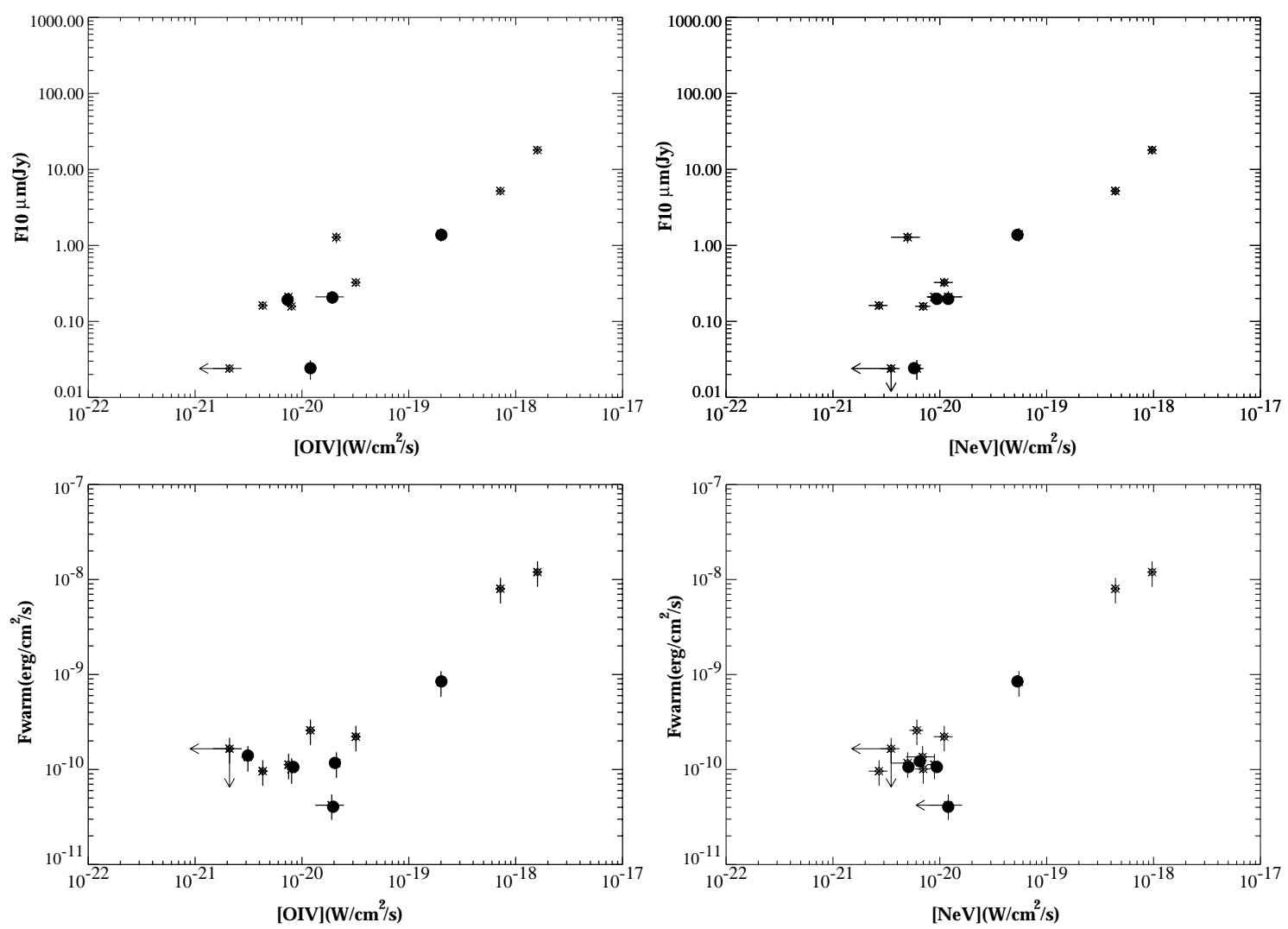

Fig. 1. The $10 \mu \mathrm{m}$ continuum flux and the so-called IR warm flux as derived by Perez-Garcia et al. (2000) are respectively compared with the $[\mathrm{O}$ IV] $] \lambda 26.9 \mu \mathrm{m}$ and $[\mathrm{Ne} \mathrm{V}] \lambda 14.3 \mu \mathrm{m}$ line fluxes for the sample of galaxies analyzed. Errors bars for the continuum and line fluxes are plotted. In some cases the errors are smaller than the symbols used, e.g., the $10 \mu \mathrm{m}$ fluxes. Seyfert 1 objects are indicated with filled symbols, all the others are Seyfert 2's.

with a progressive degradation of the coronal line - IR flux correlations as we move towards longer IR wavelengths.

The analysis of the IR spectral energy distribution of the CfA sample of Seyfert galaxies by Pérez García \& Rodríguez Espinosa (2001; cf. Sect. 1, this work) shows the existence of a separate warm dust component emission peaking at about $16 \mu \mathrm{m}$, which it is argued to be associated with the nuclear emission. Considering the above correlations, a tight relation between the flux from that warm component and the coronal emission should also be expected. This is shown in Fig. 1 (bottom panels). The linear correlation coefficients in this case are $r=0.85$ and $r=0.90$ for the comparison with the $[\mathrm{Ne} \mathrm{V}]$ and the [OIV] line fluxes respectively, the significance level being larger than $99.9 \%$.

Finally, the re-distribution of the objects in the above diagrams when the factor distance is considered is illustrated in Fig. 3. This figure shows a selection of the previous diagrams in luminosity plots, namely the comparison between the IR continuum luminosities - from 10 to $120 \mu \mathrm{m}$ - and the [OIV] $\lambda 25.9 \mu \mathrm{m}$ line luminosity for all the galaxies analyzed. In this case, the brightest objects in the sample, namely NGC 1068, Circinus and YGC 4151, show luminosities comparable to the average values in the sample. NGC 1068 shows however the largest fluxes and luminosities in all cases. Regarding the distribution of objects according to their Seyfert type, the five Seyfert type 1 galaxies in the sample (NGC 4151, NGC 5548, Mrk 335, NGC 5033 and Mrk 817) show luminosities in both the continuum and the line comparable to those measured in the Seyfert 2 group. In all the comparisons (Figs. 1 to 3 ), the two groups are well mixed and follow the same overall trend.

Figure 3 shows the same type of correlations as illustrated in the flux diagrams. The corresponding linear correlation coefficients for the comparisons involving both $[\mathrm{OIV}]$ and $[\mathrm{NeV}]$ are provided in Table 1 . As expected, the strongest correlation appears between the $10 \mu \mathrm{m}$ and the coronal line luminosities. The progressive degradation of the correlations with increasing IR wavelength is now very clearly seen in luminosity plots (Fig. 3 and Table 1). Note for instance the jump in correlation coefficient between the 25 and the $60 \mu \mathrm{m}$ quantities.

\section{Discussion}

Perez García \& Rodríguez Espinosa (2001) showed that the mid and far IR emission in Seyfert galaxies arise in two different galactic environments, namely, the nuclear region (circumnuclear star forming regions and the active nucleus) and the disk of the galaxy respectively. The present results add further support to this scenario. 

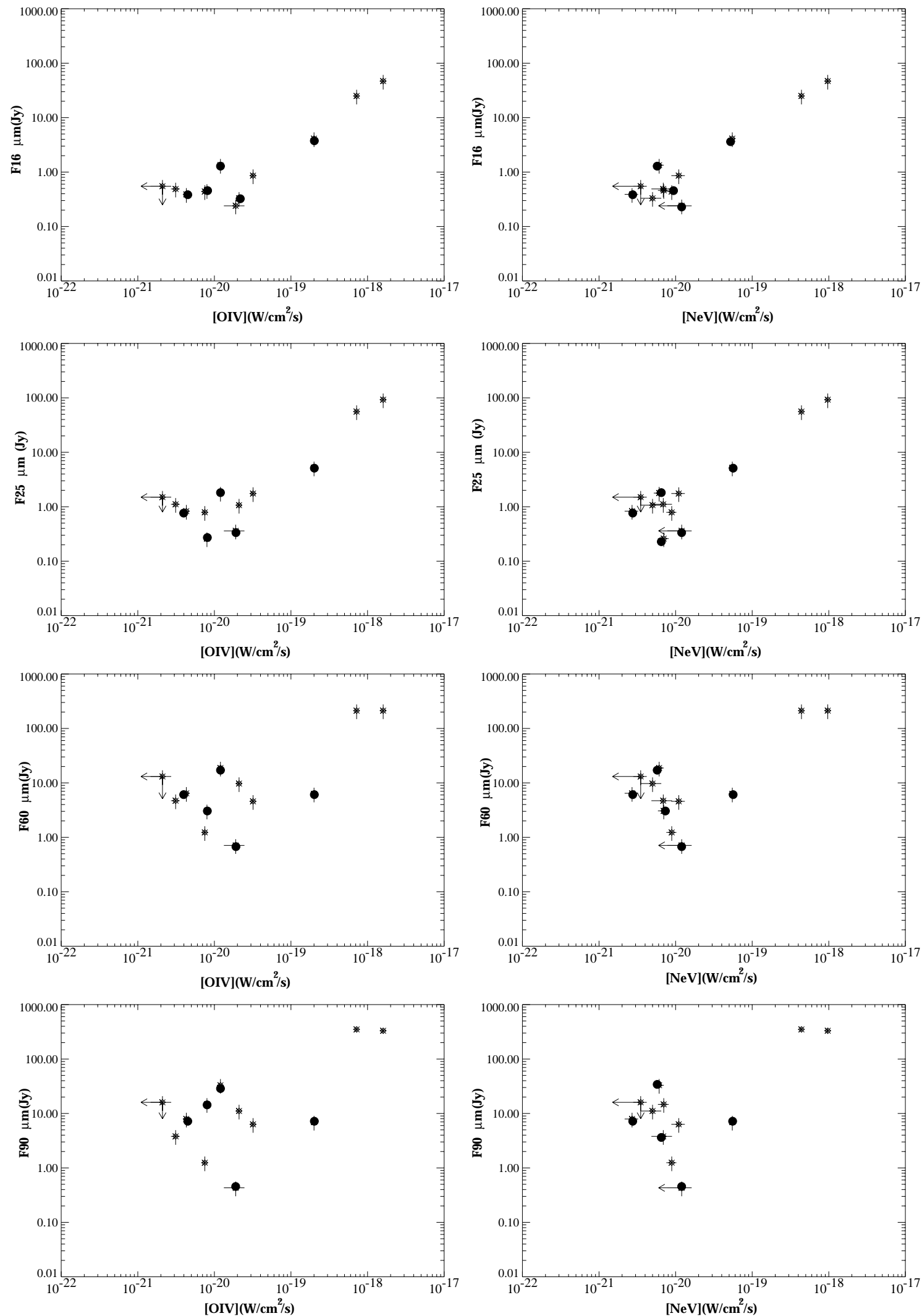

Fig. 2. The same as in Fig. 1 but involving now the comparisons with the ISO 16, 25, 60 and $90 \mu$ m fluxes.

Because the coronal lines are directly linked to the nuclear activity, the very good correlation found between the mid-IR continuum and the coronal line fluxes indicates that the mid IR continuum emission in Seyfert galaxies, regardless of their type, is largely dominated by the active nucleus. Some scatter is present in the diagrams but 

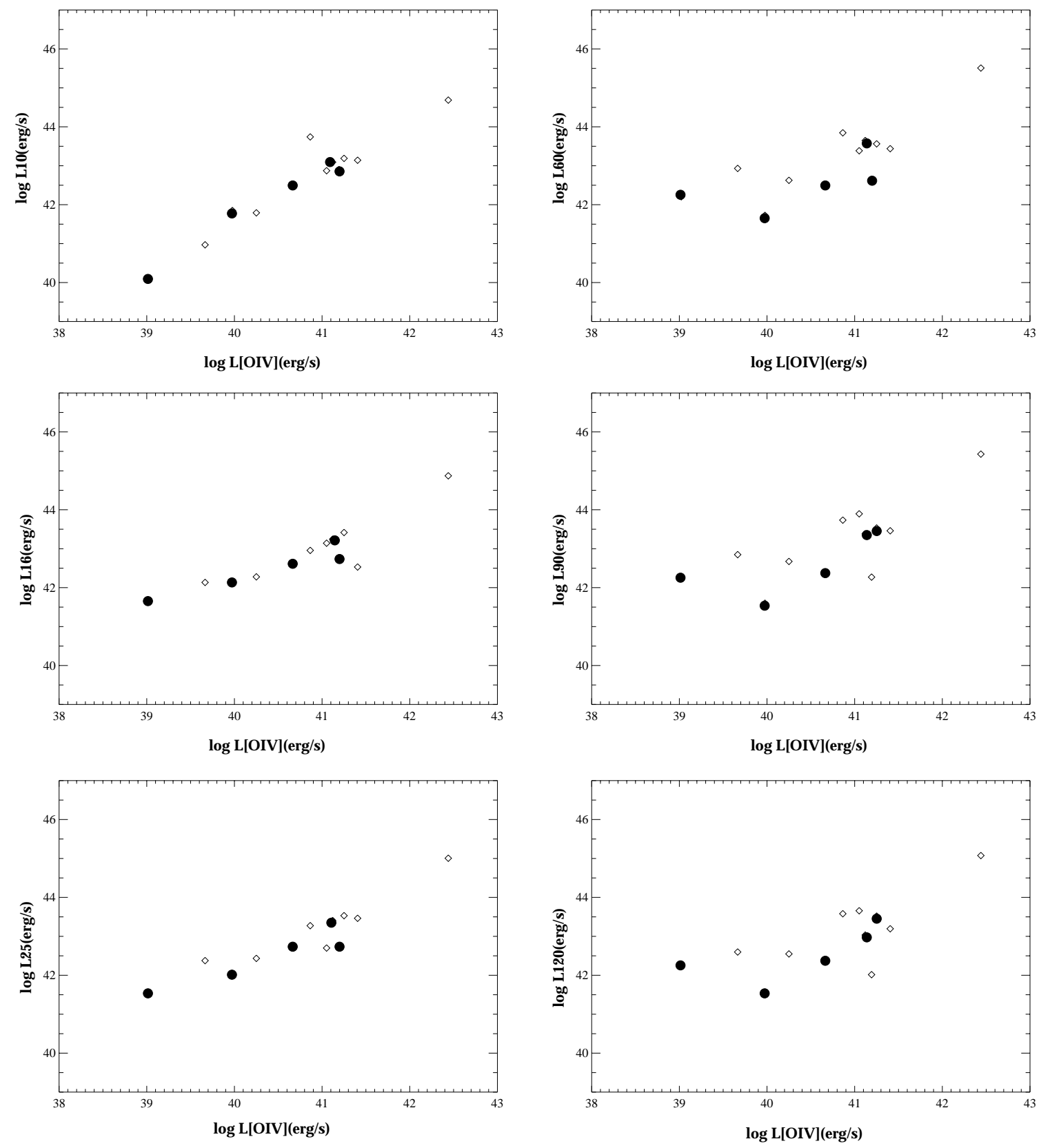

Fig. 3. The 10, 16, 25, 60, 90 and $120 \mu \mathrm{m}$ luminosities versus the [OIV] $\lambda 25.9 \mu \mathrm{m}$ luminosity for all the galaxies analyzed. Symbols are as in Fig. 1.

Table 1. Linear correlation coefficients for the comparisons between the IR continuum luminosities and the coronal line luminosities.

\begin{tabular}{ccc}
\hline & $L[\mathrm{OIV}]$ & $L[\mathrm{NeV}]$ \\
\hline \hline L10 & 0.96 & 0.95 \\
L16 & 0.85 & 0.90 \\
L25 & 0.92 & 0.87 \\
L60 & 0.80 & 0.74 \\
L90 & 0.75 & 0.69 \\
L120 & 0.73 & 0.64 \\
\hline
\end{tabular}

this is expected to be largely due to emission from circumnuclear star forming regions which contribution would be included in the large ISO apertures. In particular, the significantly better correlation with the small aperture $10 \mu \mathrm{m}$ flux might certainly be a direct consequence of the smaller aperture size used in the ground based observations.

Regarding the far IR emission, it is seen that the correlation between the coronal line emission and the mid and far IR fluxes progressively gets poorer beyond $25 \mu \mathrm{m}$ and longwards. This trend calls for a different origin for the colder component, most probably unrelated with the nuclear activity.

The most plausible source for the emission in the aforementioned IR components is dust; in the case of the midIR, or the so-called warm component, the correlation with the coronal line emission indicates that gas and dust are 
well mixed in the nuclear environment and very likely have comparable efficiencies in reprocessing the primary radiation emerging from near the central engine (see also Rudy 1984).

In the case of the far-IR, or the so-called cold component, the lack of correlation with the coronal line emission points to a different origin for the emission in that region. Perez García et al. (2000) show that the $90 \mu \mathrm{m}$ emission from the four Seyfert galaxies analyzed in their work (NGC 5033 is the only source in common with our sample) is extended, with sizes comparable to those seen in optical images. In that case, the most probable source of the emission is dust heated by the stellar population in the disks of these galaxies. If that is the general case for Seyfert galaxies as a class, no obvious relation between the far-IR continuum and the coronal line emission should be expected, in agreement with the result found in this work.

\section{References}

Contini, M., Prieto, M. A., \& Viegas, S. M. 1999, ApJ, 505, 621

Edelson, R. A., Malkan, M. A., \& Rieke, G. H. 1987, ApJ, 321, 233
Edelson, R. A. 1978, ApJ, 226, 550

Genzel, R., et al. 1998, ApJ, 498, 579

Giuricin, G., Mardirossian, F., \& Mezzetti, M. 1995, ApJ, 446, 550

Krabbe, A., et al. 1997, ApJ, 476, 98

Maiolino, R., Ruiz, M., Rieke, G. H., \& Keller, L. D. 1995, ApJ, 446, 561

Maiolino, R., Krabbe, A., Thatte, N., \& Genzel, R. 1998, ApJ, 493, 650

Moorwood, A., et al. 1996, A\&A, 315, L109

Pérez García, A. M., \& Rodríguez Espinosa, J. M. 2001, ApJ, in press

Pérez García, A. M., Rodríguez Espinosa, J. M., \& Fuensalida, J. J. 2000, ApJ, 529, 875

Pérez García, A. M. Rodríguez Espinosa, J. M., \& Santolaya Rey 1998, ApJ, 500, 685

Prieto, M. A., \& Viegas, S. M. 2000, ApJ, 252, 238

Rieke, G. H., \& Lebofsky, M. J. 1978, ApJ, 222, 49

Rigopoulou, D., Papadakis, L., Lawrence, A., \& Ward, M. 1997, A\&A, 327, 493

Rodríguez Espinosa, J. M., \& Pérez García, A. M. 1997, ApJ, 487, L33

Rodríguez Espinosa, J. M., Rudy, R. J., \& Jones, B. 1986, ApJ, 309, 76

Rudy, R. J. 1984, ApJ, 284, 33 